\title{
CHARACTERIZATION OF SPINNING ROTOR GAUGE-3 USING ORIFIC FLOW SYSTEM AND STATIC EXPANSION SYSTEM
}

\author{
Ashok Kumar ${ }^{1,2, a}$, Vikas N. Thakur ${ }^{1,2 b}$, Harish $\operatorname{Kumar}^{1, \mathrm{c}}$ \\ ${ }^{1}$ CSIR-National Physical Laboratory, Dr. K. S. Krishnan Marg, Delhi 110012, India \\ 2 Academy of Scientific and Innovative Research (AcSIR), Ghaziabad-201002, India \\ a ashok553@nplindia.org, b vikasnthakur92@gmail.com, ${ }^{\mathrm{c}}$ harish@nplindia.org
}

\begin{abstract}
:
This article elucidates the calibration of newly procured spinning rotor gauge (SRG 3) from MKS Instruments, USA using primary vacuum standards: Orifice flow system (OFS) and Static expansion system (SES) established at National Physical Laboratory, India (NPLI) in the range of $10^{-4} \mathrm{~Pa}$ to $1 \mathrm{~Pa}$ and further compared with manufacturer reported value which was calibrated by transfer standard of National Institute of Standards \& Technology (NIST). The key parameters to calculate the pressure measured by SRG is the accommodation coefficients. The accommodation coefficients for $\mathrm{N}_{2}$ gas obtained using OFS, SES, and calibration report form NIST USA (SRG2) are $0.957,0.961$, and 0.954 respectively.
\end{abstract}

Keywords: Vacuum; orifice flow system; static expansion system; Pressure; spinning rotor gauge

\section{INTRODUCTION}

As a national metrology institute, NPLI has the responsibility of maintaining the various primary vacuum standards and disseminate the traceability down to secondary and reference standards. Barometric Pressure and Vacuum metrology section maintained the pressure standards in the range of 1 micro $\mathrm{Pa}$ to $360 \mathrm{kPa}$. Among these standards, this section has two primary vacuum standards OFS and SES which measure the pressure in the range of 0.1 $\mathrm{Pa}$ to $1 \mu \mathrm{Pa}$ and $0.05 \mathrm{~Pa}$ to $10 \mathrm{~Pa}$, respectively with associate uncertainty $2 \%$ and $0.4 \%$ of the pressure reading at $\mathrm{k}=2$. The vacuum primary standards have participated in the international key comparisons $[1,2]$ and found the reputations at the global level. There are many other transfer standards like resonant silicon gauge (RSG), old spinning rotor gauge (SRG 2), capacitance diaphragm gauge (CDGs), etc. which are frequently calibrated by SES and OFS. SES and OFS are two primary pressure standards which are used to generate pressures very accurately and sitting at the top of traceability chain in vacuum metrology in India. There are some defense laboratories, research institutions and accredited laboratories which also possess CDGs and SRGs as transfer or secondary standards which need to be calibrated time to time using NPLI primary vacuum standards. The low precision ionization gauges were used as transfer standard for inter-comparisons before high precision SRGs and CDGs came into the existence. Many intercomparisons were performed previously using these two vacuum standards i.e. OFS and SES [3,4].

Recently this section bought SRG 3 which has pressure measuring range of $1 \mathrm{~Pa}$ to $10^{-4} \mathrm{~Pa}$. Comsa et. al first invented the spinning rotor gauge in vacuum and high vacuum range with unmatched accuracy among the existing transfer standards, almost similar to the uncertainty of OFS [5]. SRG is most commonly used as transfer vacuum standard since for the first time it was used as transfer standard by Messer et. al. [6]. In 1989 and 2014, the international key comparisons were performed using two SRGs [7]. MKS has designed and developed the third generation SRG and named it SRG3 which is more robust and stable in high vacuum range. The NPLI studied the accommodation factors of SRG3 in details. For the same purpose, NPLI has calibrated the SRG3 using both the primary standards (OFS and SES) and further compared with the value given by SRG2 (transfer standard of NIST)

\section{APPARATUS WITH CHARACTERIZATION DETAILS}

Static expansion system consists of two large chambers of different volumes of $V_{S}=241$ and $V_{L}=721$. The gauge under calibration (GUC) connected to the port of the chamber of volume 721 . Schematic diagram of SES is shown in Figure 1. There are two larger chambers as discussed above are mechanically connected with three small chambers of volume $v_{1}=25 \mathrm{ml}, v_{2}=25 \mathrm{ml}$ and $v=343 \mathrm{ml}$ between the valves 1 and 2, 4 and 5 and 
2 and 3, respectively as shown in Figure 1 . The large chamber $V_{L}$ connected to a turbomolecular pump having automated pneumatic gate valve to control and release the generate pressure. The chamber $V_{s}$ was pumped through a separate pumping system. Prior to any measurement, the whole system was backed at $200{ }^{\circ} \mathrm{C}$ and minimum $10^{-8} \mathrm{~Pa}$ for several days while system connected to pumping stations/systems. The pressures were generated by following the expansion equation: $v_{2} \rightarrow v_{2}+V_{L}$. The principle of working of SES is the static expansion of gas. The gas is first enclosed in the smaller volume i.e. $25 \mathrm{ml}$, then it is allowed to enter the larger volume i.e. 721 to expand under nearly perfect isothermal conditions. The pressure in the larger volume was calculated using the gas equation given by equation (1),

$$
P_{L}=\frac{P_{S} T_{L}}{R T_{S}\left(1+P_{S} B^{\prime \prime}\right)}
$$

where $R=\frac{V_{S}+V_{L}}{V_{S}}$ is the ratio of final volume to initial volume, $P_{S}$ and $P_{L}$ are the initial and final pressure in smaller volume and larger volume respectively, $V_{S}$ and $V_{L}$ are the volumes of smaller and larger chambers respectively, $T_{S}$ and $T_{L}$ are the temperature values in the smaller and larger chambers respectively and $B^{\prime \prime}=B / T R_{m}$ is the second virial coefficient, $R_{m}$ is the molar gas constant. SES has the pressure measuring range of $0.05 \mathrm{~Pa}$ to $10 \mathrm{~Pa}$ with the expanded uncertainty of $0.4 \%$ of pressure reading at $k=2$.

Orifice flow system is another vacuums standard which is used to calibrate the vacuum gauges in the molecular flow region i.e. the molecular mean free path is larger than the dimension of the vessel. OFS consists of two chambers of equal volume connected by a cylindrical path. The schematic diagram of the OFS is shown in Figure 2(a). Both the chambers are of equal diameter of $30 \mathrm{~cm}$. The gauges under test are to be connected to the upper chamber. The residual gas analyzer was connected to the upper chamber to check the purity of the gas in the chamber. The configuration of the experimental setup consists of two spheres to have the maximum isotropy of the molecular distribution of Maxwellian velocity distribution. The chambers were pumped out by following the same procedure as used explained by Hojo et. al [9]. The turbomolecular pump is used for pumping the gas inside the chamber. A titanium sublimation pump (TSP) was also connected to the lower chamber. Before the calibration start, the chambers were baked for several days by heating tapes and an ultimum vacuum of $10^{-10} \mathrm{~Pa}$ was maintained. The temperatures were measured by the platinum resistance thermometers (PRTs) connected five different points in the system. The average of the temperatures in the calibration sphere was used as the reference temperature.

At the centre of the cylinder path a disk is placed made up of copper known as orifice denoted by ' $\mathrm{C}$ '. The magnified view of orifice is shown in Figure 2(b). The orifice has the diameter of $10 \mathrm{~mm}$. The edges of the aperture are prepared

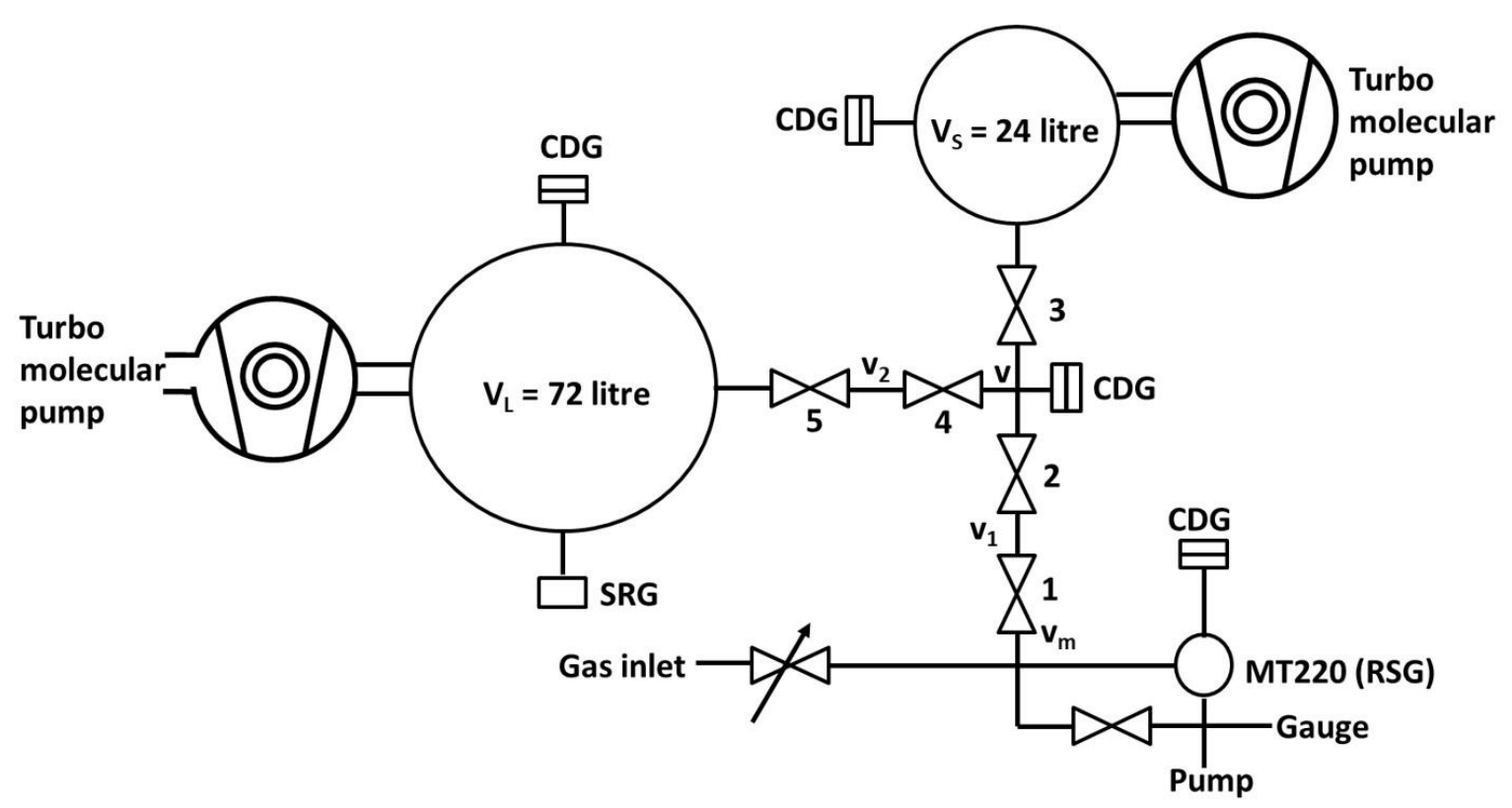

Figure 1: Schematic of static expansion system having chambers of volume 721 and 24 1. [8]. 


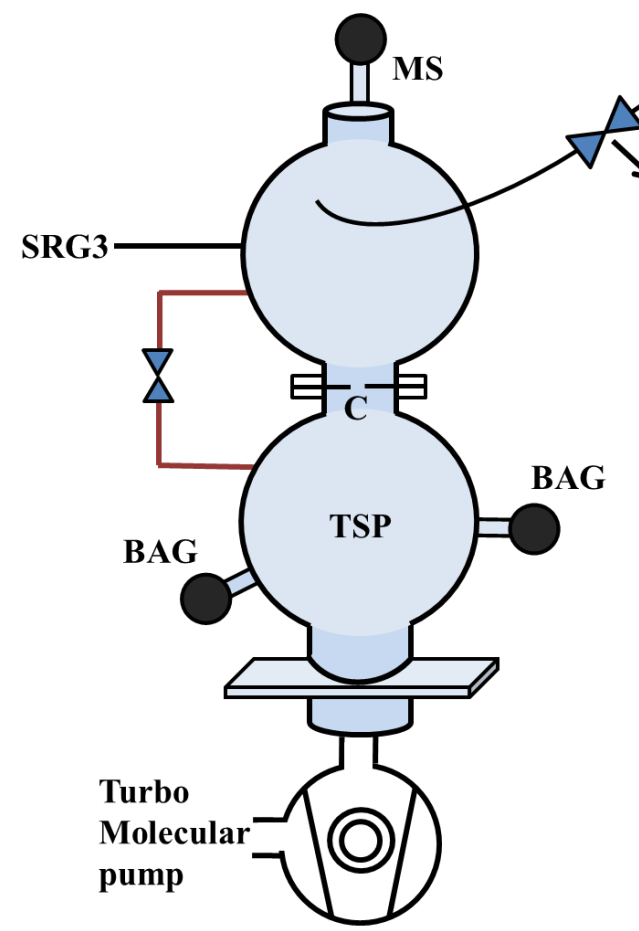

(a)

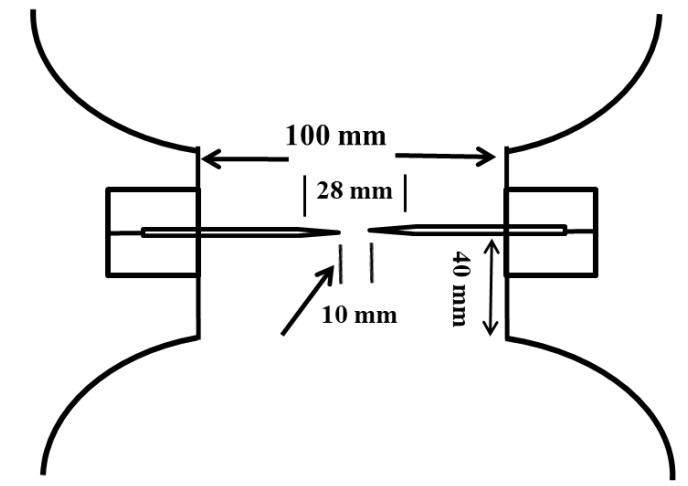

(b)

Figure 2: (a) Schematic diagram of the orifice flow system [8], TSP - titanium sublimation pump, BAG - nude Bayard Alpert gauges, MS - mass spectrometer, C- orifice (b) magnified view of the orifice.

in such a manner that the thickness of the disk at the periphery of the orifice nearly approached to zero. The working principle of the OFS is based on the flow of gas in molecular flow regions through the orifice of known conductance (C) connected across two chambers [10]. If $\mathrm{Q}$ is the flow rate of the gas throughout then the pressure upstream is given by equation (2)

$$
P=\frac{Q}{C}\left(1+\frac{C}{S_{P}}\right)
$$

where $P$ is the pressure upstream of the orifice or the pressure in the calibration chamber and $\mathrm{Sp}$ is the pumping speed available in the chamber below the orifice.

The SRG 3 consists of ball-tube assembly using which vacuum is to be measured. The ball spins using a controlled electronics mechanism with the frequency of nearly $450 \mathrm{~Hz}$. While spinning the ball slows down due to the gas friction and falls down with the gas pressure. A sensing head is mounted on the ball-tube housing to keep the ball immobilized and collect the deceleration of ball due to change in pressure inside the chamber. According to the basic pressure measurement method of SRG 3, the relation between gas pressure $\mathrm{P}$ and the speed of the ball is given by equation (3) [11]

$P=\left(-\frac{d \omega}{d \omega t}\right) \frac{a \rho}{10 \sigma} \sqrt{\frac{8 \pi R_{m} T}{M}}-[R D]$.
Here, $a$ and $\rho$ are the radius and density of the SRG rotor, respectively. $\mathrm{M}$ and $\mathrm{T}$ are the molecular mass of the gas and temperature inside the chamber, RD is the residual drag, i.e., the deceleration of the rotor, and $-\frac{d \omega}{d \omega t}$ is the relative deceleration rate of the sphere per unit time and has units of $\mathrm{s}^{-1}, \sigma$ is the accommodation coefficient, and $\mathrm{R}_{\mathrm{m}}$ is molecular gas constant, $8.314 \times 10^{3}(\mathrm{Nm} / \mathrm{kmol} \cdot \mathrm{K})$.

\section{RESULTS \& DISCUSSION}

The SRG3 ball was placed in their respective tubular housing. Since the comparison did not involve transportation of the ball/tube assemblies to other laboratories, no need was felt of using the transport device for fixing the balls in the tubular housings during transportation. However, the ball/tube assembly was fitted with a mini ultra-high vacuum (UHV) right angle valve so that the rotors could be isolated from exposure to air during venting of the chamber for shifting the ball/tube assemblies from one calibration system to the other and back. Precautions were taken to keep the sensing head mounted on the tubular housing so as to keep the ball immobilized because of permanent magnets of the head, while transferring the ball/tube assembly from one system to the other.

The SRG3 was first calibrated on the SES, in the pressure range of $0.1-1 \mathrm{~Pa}$, by following the method of SES i.e. successive expansion of the gas from one chamber to another. Later, it was calibrated using OFS in the range of $0.001-0.01$ 
Pa. The key parameter to compare, calibrate, and characterize any SRG is accommodation coefficients. The accommodation coefficient is obtained by the intercept of the plot of the calibration factor - pressure measured by the vacuum standards (i.e. SES and OFS), where calibration factor is given by the ratio of the pressures measured by gauge under calibration (i.e. SRG3) and vacuum standards. As SRG3 has measuring range of $10^{-4} \mathrm{~Pa}$ to $1 \mathrm{~Pa}$, therefore, SRG3 was calibrated by both SES and OFS for vacuum and high vacuum range, respectively. To calculate the value of accommodation coefficients, the calibration factor for SES and OFS are plotted as a function of pressure in Figure 3 and Figure 4, respectively. The y-intercept (at zero pressure) of the graphs in Fig. 3 and Fig. 4 give the accommodation coefficients of the SRG3 measured by SES and OFS, respectively. The values of accommodation coefficients calculated using SES, OFS and SRG2 (MKS, NIST) are given by Table 1.

The values of accommodation coefficients given in Table 1 show the maximum standard deviation of $\approx 0.003$.

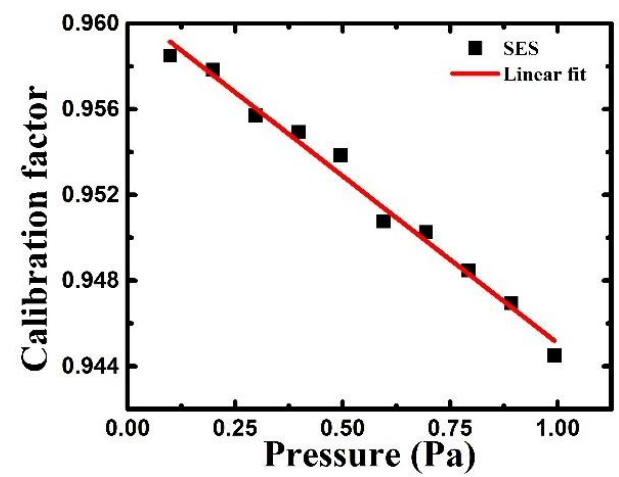

Figure 3: Calibration factor of SRG3 as a function of pressure using the SES.

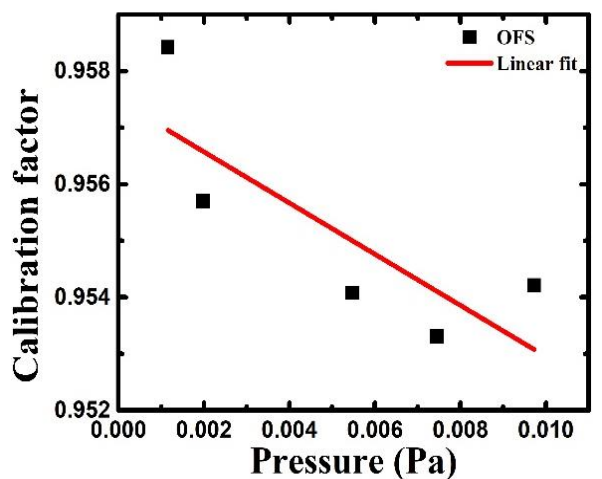

Figure 4: Calibration factor of SRG3 as a function of pressure using the OFS.

Table 1: Accommodation coefficients obtained by different vacuum standards.

\begin{tabular}{|c|l|c|}
\hline S. No. & Standard & $\begin{array}{l}\text { Accommodation } \\
\text { coefficient }\end{array}$ \\
\hline 1 & SES & 0.961 \\
\hline 2 & OFS & 0.957 \\
\hline 3 & SRG2 (MKS, NIST) & 0.954 \\
\hline
\end{tabular}

\section{SUMMARY}

The values of the accommodation coefficients were successfully calculated and the values obtained from SES and OFS were further compared with the value obtained from SRG3 (MKS, NIST). It is found that these three values of accommodation coefficients having the maximum standard deviation of $\approx 0.003$.

\section{REFERENCES}

[1] D.A. Olson, P.J. Abbott, K. Jousten, F.J. Redgrave, P. Mohan, S.S. Hong, Final report of key comparison CCM.P-K3: absolute pressure measurements in gas from $3 \times 10-6 \mathrm{~Pa}$ to $9 \times 10$ -4 Pa, Metrologia. 47, 07004, 2010.

[2] K. Jousten, K. Arai, U. Becker, O. Bodnar, F. Boineau, J.A. Fedchak, V. Gorobey, W. Jian, D. Mari, P. Mohan, J. Setina, B. Toman, M. Vičar, Y.H. Yan, Final report of key comparison CCM.PK12 for very low helium flow rates (leak rates), Metrologia 50, 07001, 2013.

[3] G. Reich, C. Meinke, Comparison between static and dynamic calibration methods for ionization gauges, Vacuum 17(3), 164, 1967.

[4] K.F. Poulter, A. Calcatelli, P.S. Choumoff, B. Iapteff, G. Messer, G. Grosse, Intercomparison of vacuum standards of countries within the European community in the range $8 \times 10-5$ to $8 \times 10-2 \mathrm{~Pa}$., J. Vac. Sci. Technol. 17, 679-687, 1980.

[5] G. Comsa, J. K. Fremerey, and B. Lindenau, in Proceedings of the 8th International Vacuum Congress, Cannes, Le Vide, 1980.

[6] G. Messer, P. Röhl, G. Grosse, W. Jitschin, High vacuum measured by the spinning rotor gauge: Status report of the Bureau International des Poids et Mesures intercomparison, J. Vac. Sci. Technol. A Vacuum, Surfaces, Film. 5, 2440-2443, 1987.

[7] J.K.N. Sharma, P. Mohan, W. Jitschin, P. Röhl, Intercomparison of vacuum standards between the Physikalisch Technische Bundesanstalt (Germany) and the National Physical Laboratory (India) using two spinning rotor gauges, J. Vac. Sci. Technol. A Vacuum, Surfaces, Film. 7(4), 2788-2793, 1989.

[8] J.K.N. Sharma, P. Mohan, D.R. Sharma, Comparison of two primary pressure standards using spinning rotor gauges, J. Vac. Sci. Technol. A Vacuum, Surfaces, Film. 8(2), 941-947, 1990.

[9] H. Hojo, M. Ono, and K. Nakayama, in Proceedings of the 7th Interna. tional Vacuum Congress and 3rd International Conference Solid Surfaces. Vienna, 1977 (Berger and Sohne, Horne, Austria), p. 117, 1977.

[10] P. Mohan, Vacuum gauge calibration at the NPL (India) using orifice flow method, Vacuum 51(1), 69-74, 1998.

[11] P. Mohan and H. Kumar, Static expansion primary vacuum standard - Part 2: Characterization of two spinning rotor gauges, Mapan-J. Metrol. Soc. India. 24, 111-118, 2009. 